\title{
Direct method for deriving equilibrium equations in solid continuous systems
}

\author{
Mohammad Asadi Dalir* and Rahman Seifi
}

Mechanical Engineering Department, Bu-Ali Sina University, 65175-4161 Hamedan, Iran

\begin{tabular}{|c|c|}
\hline ARTICLE INFO & A B S T RAC T \\
\hline $\begin{array}{l}\text { Article history: } \\
\text { Received March 6, } 2014 \\
\text { Accepted } 23 \text { August } 2014 \\
\text { Available online } \\
\text { 15 October } 2014 \\
\text { Keywords: } \\
\text { Equilibrium equations } \\
\text { Continuous systems } \\
\text { Direct method } \\
\text { Intensive properties }\end{array}$ & $\begin{array}{l}\text { In this paper, a simplified method is proposed for deriving equilibrium equations in continuous } \\
\text { systems. The new method is indeed the direct applying of Newton's laws on free body diagram } \\
\text { of point. First, by describing the concept of equilibrium equations and investigating the } \\
\text { differences between concentrated masses and continuous systems, the physical basis of new } \\
\text { method is introduced. It is shown that, using intensive properties simplifies the analysis of } \\
\text { continuous systems. For verifying the new method, the governing equations in Cartesian, polar } \\
\text { and spherical coordinates systems are derived. We have to consider nonlinear terms due to } \\
\text { developing large slopes in system. Hence, nonlinear governing equations in Cartesian system } \\
\text { are derived too. Finally by noting to the simplicity of new method and its independency from } \\
\text { complicated differential and vector analysis in other methods such as Hamiltonian and classic } \\
\text { methods, the interests of new method are emphasized. By knowing concept of physical point, a } \\
\text { united process is accessible which is extendable to other governing equations of continuous } \\
\text { systems. }\end{array}$ \\
\hline
\end{tabular}

(c) 2014 Growing Science Ltd. All rights reserved.

\section{Introduction}

The study of moving objects and their behavior analysis under external factors such as forces and moments is simply possible by using the triple laws of Newton. However, all of the problems in mechanical science are not including the concentrated masses. For investigating the behavior of mechanical structures and fluids motion, it is necessary to consider separately all of the constituent particles of material. On the other hand, the mathematical model of each particle is considered as a point and any continuous system includes infinite number of points. Herein, the simplest method that can be applied is writing of equations of motion for each particle separately. The most important problem in this way is that, point is a mathematical definition and has no physical interpretation. Although, the dimensions of each particle is so many infinitesimal, but it cannot be assumed as a point. Because, a physical particle has mass and occupies a certain volume, while, the point has not any dimension. So, modeling of a particle as a point seems to be impossible from physical point of view.

* Corresponding author. Tel.: +988118292630

E-mail addresses: mh.dalir@yahoo.com (M. Asadi Dalir) 
On the other hand, a continuous system never can be analyzed by writing the equation of motions for the particles with specified volume. In such case, two major problems will occur. The first is that in study of continuous systems we investigate all particles in the system. While, a physical particle has dimensions and is made by smaller particles. So, study of the internal points in considered particle is impossible. The second problem is arisen from the first one. In such case, the response of system will be modeled as a discrete function which involving discontinuity in the boundary of considered particle. Both of mentioned problems have been overcome by the aid of mathematics. If a particle with specified dimensions be studied, by tending its dimensions to zero the point is achieved. While as mentioned, the point is a mathematical definition and finding a physical imagination for point is a complicated matter and has been remained an unresolved issue yet.

In this paper, the governing equations in continuous systems are investigated from a conceptual point of view. For this, the principle and basics of a simple method has been expressed. Firstly to this end, it is shown that having a suitable understanding about concept of governing equations in continuous systems can simplify the investigation of these systems. Thus using intensive properties is proposed. Finally, the equilibrium equations are derived by using the new method in rectilinear and curvilinear coordinates systems. Beside simplicity of new method, this method has made possible the understanding of governing equations concept for any researcher due to its conceptual point of view. The method has also a general interpretation for point, and is extendable to other equations in continuous systems. This matter is leaded to a united form be achieved, for extraction of governing equations in continuous systems from their corresponding model of solid objects.

\section{Formulation}

For review of the systems which involving concentrated masses (mass-spring), governing equations are extracted by considering applied forces in free body diagram. While, when we investigate motion of solid continuous systems, governing equations are versus stress components. On the other hand, Hook's law for concentrated masses is the relationship between force and deformation, while this law for solid continuous systems is the relationship between stresses and strains.

The note which here we are going to explain is that, in continuous systems equations cannot be achieved versus forces with unite of “ $\mathrm{N}$ ” $\left(\mathrm{Kg} . \mathrm{m} / \mathrm{s}^{2}\right)$. Hook's law in these systems is not explainable as a relationship between force and deformation too. Both of these limitations have a common reason which can be found in the difference of physical element and mathematical point as reviewed in pervious section. Consider a concentrated force (with unite of "N") which is applied on a specified area. To finding effect of this force on mentioned area, this effect should be studied on all of its constituent points. While force is an extensive property, cannot be applied on a point. But if an intensive property be accessible, it is simply extensible to all points. Surface force or stress is same as extensive force which has been converted to an intensive property by writing it per unite area. Consequently, Hook's law also in continuous systems is indeed versus forces, which are converted to intensive properties. Thus, in solid continuous systems there are some extensive forces which are the potential of stress components. These forces can be defined as: $\sigma_{i j}=\partial F_{i j} / \partial A\left(x_{k} x_{j}\right)$. In this equation $i$ is outward normal unite vector direction of surface $A, j$ is force direction and $x_{j}$ and $x_{k}$ are in-plane directions of $A$.

Regarding to above context, our propose is that, for deriving motion equations in continuous systems, the most appropriate way is using from intensive properties. As any extensive property depends on dimensions but each point has no dimension; so, extensive properties and point are inconsistent with each other. Thus, using from intensive properties lead to overcoming the problem. In order to achieving equations versus intensive properties, they can be written per unit volume. 
Consider the Newton's second law as a governing equation versus extensive properties which is a function of dimensions: $\sum F-m a=G(V)=0$. To converting this equation versus intensive properties it can be divided per volume and for generalizing it to each arbitrary point, the considered volume should approaches to zero:

$\lim _{\Delta V \rightarrow 0} \frac{\Delta G(V)}{\Delta V}=\frac{d G(V)}{d V}=0$

where $\Delta G(V)$ is same as $G(V)$ which describes the motion of a particle with dimensions $\Delta V$. For converting governing equations to functions versus intensive properties it is sufficient that extensive properties be differentiated with respect to triple orthogonal directions in any arbitrary coordinates system. In Cartesian coordinates systems, derivative with respect to orthogonal directions is equal to volume derivative as is shown in Eq. (1). But in curvilinear coordinates systems, $d V=\psi d x_{i} d x_{j} d x_{k}$, where, $\psi$ is equal by $r$ and $r^{2} \sin \theta$ in polar and spherical coordinates, respectively.

Here our purpose is the review of equilibrium equations, so $G(V)$ is the second law of Newton. Eq. (1) can cover other governing equations in continuous systems too. We note that, in a solid continuous system, existent forces are resultant of body forces due to acceleration and potential forces which give the stress components $\left(\sum F=F_{i j}+F_{j}\right)$. Thus Newton's second law can be replaced in Eq. (1) which gives:

$F_{i j, k j i}+F_{j, k j i}-\rho a \psi=0$

where, as mentioned $F_{i j}$ are the available forces in any arbitrary point of solid continuous systems which give the components of $\sigma_{i j}$. $\rho$ is density, $a$ is acceleration and $F_{j}$ are body forces. The components of $i, j$ and $k$ are the orthogonal directions in any arbitrary coordinates system. As in Eq. (2) is seen, derivative with respect to direction of $i$ (direction of surface normal unite vector) should be the last one operator which is applied. Because, available governing equations are versus stress components (Saad, 2005), for verifying the method; Eq. (2) should be arranged in a way that give the governing equations versus stress components too. Thus, the derivative operator should be applied in that way which, the components of $F_{i j}$ can be converted to stress components, then the third operator be applied. Whereas Eq. (2) itself is the general form of governing equations in solid continuous systems. No matter to be either as a function of stress components or potential forces. In Eq. (2) available forces in each point are converted to body forces and their effects is proportional by extent of considered body. So, their effects on each point are exactly proportional with its dimensionless nature.

\section{Derivation of governing equations in continuous systems}

In this section for verifying Eq. (2), the governing equations of solid continuous systems in Cartesian, polar and spherical coordinates will be derived.

\subsection{Equilibrium equations in Cartesian coordinates system}

Stress component in Cartesian coordinates systems, which are induced by applied potential forces are as following (Fig. 1):

$$
\begin{array}{llll}
\sigma_{x x}=\frac{\partial^{2} F_{x x}}{\partial y \partial z} & \sigma_{y x}=\frac{\partial^{2} F_{y x}}{\partial x \partial z} & \sigma_{z x}=\frac{\partial^{2} F_{z x}}{\partial y \partial x} & f_{x}=\frac{\partial^{3} F_{x}}{\partial x \partial y \partial z} \\
\sigma_{x y}=\frac{\partial^{2} F_{x y}}{\partial y \partial z} & \sigma_{y y}=\frac{\partial^{2} F_{y y}}{\partial x \partial z} & \sigma_{z y}=\frac{\partial^{2} F_{z y}}{\partial y \partial x} & f_{y}=\frac{\partial^{3} F_{y}}{\partial x \partial y \partial z}
\end{array}
$$


$\sigma_{x z}=\frac{\partial^{2} F_{x z}}{\partial y \partial z} \quad \sigma_{y z}=\frac{\partial^{2} F_{y z}}{\partial x \partial z} \quad \sigma_{z z}=\frac{\partial^{2} F_{z z}}{\partial y \partial x} \quad f_{z}=\frac{\partial^{3} F_{z}}{\partial x \partial y \partial z}$

By considering Fig. 1, the equilibrium equations in three directions based on Newton's first law are:

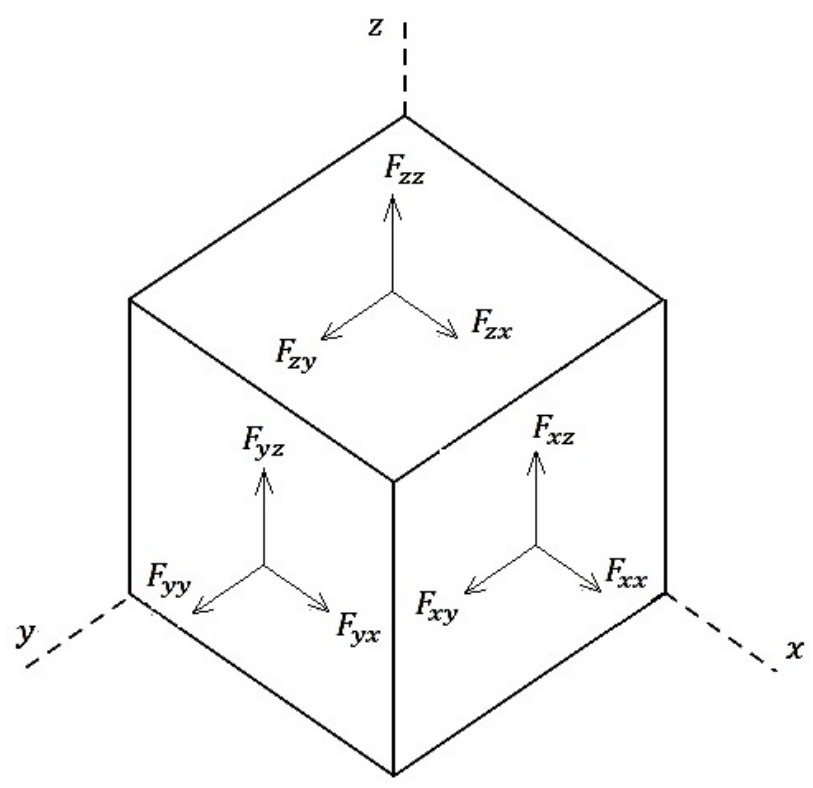

Fig. 1. The operator potential forces of solid continuous systems in Cartesian coordinates systems

$$
\begin{aligned}
& \sum \overrightarrow{P_{x}}=0 \Rightarrow F_{x x}+F_{y x}+F_{z x}+F_{x}=0 \\
& \sum \overrightarrow{P_{y}}=0 \Rightarrow F_{x y}+F_{y y}+F_{z y}+F_{y}=0 \\
& \sum \overrightarrow{P_{z}}=0 \Rightarrow F_{x z}+F_{y z}+F_{z z}+F_{z}=0
\end{aligned}
$$

The important note in Eq. (4) is that, the components of $F_{i j}$ are the available forces in any arbitrary point as a function of volume. This equation is indeed arranged for review of a point. Consequently, due to dimensionless nature of a point, it is unnecessary to be considered operating forces in all six faces of element for applying Newton's first law. Eq. (4) describes the relative motion of volume $V$ with respect to its environment. But the analysis of its internal points by this equation is impossible due to extensive nature of this equation. Hence, by replacing it in Eq. (2), the governing equations for all points are achieved:

$$
\begin{aligned}
& \frac{\partial}{\partial x}\left(\frac{\partial^{2} F_{x x}}{\partial y \partial z}\right)+\frac{\partial}{\partial y}\left(\frac{\partial^{2} F_{y x}}{\partial x \partial z}\right)+\frac{\partial}{\partial z}\left(\frac{\partial^{2} F_{z x}}{\partial x \partial y}\right)+\left(\frac{\partial^{3} F_{x}}{\partial x \partial y \partial z}\right)=0 \\
& \frac{\partial}{\partial x}\left(\frac{\partial^{2} F_{x y}}{\partial y \partial z}\right)+\frac{\partial}{\partial y}\left(\frac{\partial^{2} F_{y y}}{\partial x \partial z}\right)+\frac{\partial}{\partial z}\left(\frac{\partial^{2} F_{z y}}{\partial x \partial y}\right)+\left(\frac{\partial^{3} F_{y}}{\partial x \partial y \partial z}\right)=0 \\
& \frac{\partial}{\partial x}\left(\frac{\partial^{2} F_{x z}}{\partial y \partial z}\right)+\frac{\partial}{\partial y}\left(\frac{\partial^{2} F_{y z}}{\partial x \partial z}\right)+\frac{\partial}{\partial z}\left(\frac{\partial^{2} F_{z z}}{\partial x \partial y}\right)+\left(\frac{\partial^{3} F_{z}}{\partial x \partial y \partial z}\right)=0
\end{aligned}
$$

By inserting Eq. (3) into Eq. (5), the equilibrium equations in Cartesian coordinates system is achieved: 
$\frac{\partial \sigma_{x x}}{\partial x}+\frac{\partial \sigma_{y x}}{\partial y}+\frac{\sigma_{z x}}{\partial z}+f_{x}=0$

$\frac{\partial \sigma_{x y}}{\partial x}+\frac{\partial \sigma_{y y}}{\partial y}+\frac{\sigma_{z y}}{\partial z}+f_{y}=0$

$\frac{\partial \sigma_{x z}}{\partial x}+\frac{\partial \sigma_{y z}}{\partial y}+\frac{\sigma_{z z}}{\partial z}+f_{z}=0$

\subsection{Equilibrium equation in polar coordinates system}

The stress components in polar coordinates as depicted in Fig. 2 are as follows:

$$
\begin{aligned}
\sigma_{r r}=\frac{\partial^{2} F_{r r}}{r \partial \theta \partial z} & \sigma_{\theta r}=\frac{\partial^{2} F_{\theta r}}{\partial r \partial z} & \sigma_{z r}=\frac{\partial^{2} F_{z r}}{r \partial \theta \partial r} & f_{r}=\frac{\partial^{3} F_{r}}{r \partial r \partial \theta \partial z} \\
\sigma_{r \theta}=\frac{\partial^{2} F_{r \theta}}{r \partial \theta \partial z} & \sigma_{\theta \theta}=\frac{\partial^{2} F_{\theta \theta}}{\partial r \partial z} & \sigma_{z \theta}=\frac{\partial^{2} F_{z \theta}}{r \partial \theta \partial r} & f_{\theta}=\frac{\partial^{3} F_{\theta}}{r \partial r \partial \theta \partial z} \\
\sigma_{r z}=\frac{\partial^{2} F_{r z}}{r \partial \theta \partial z} & \sigma_{\theta z}=\frac{\partial^{2} F_{\theta z}}{\partial r \partial z} & \sigma_{z z}=\frac{\partial^{2} F_{z z}}{r \partial \theta \partial r} & f_{z}=\frac{\partial^{3} F_{z}}{r \partial r \partial \theta \partial z}
\end{aligned}
$$

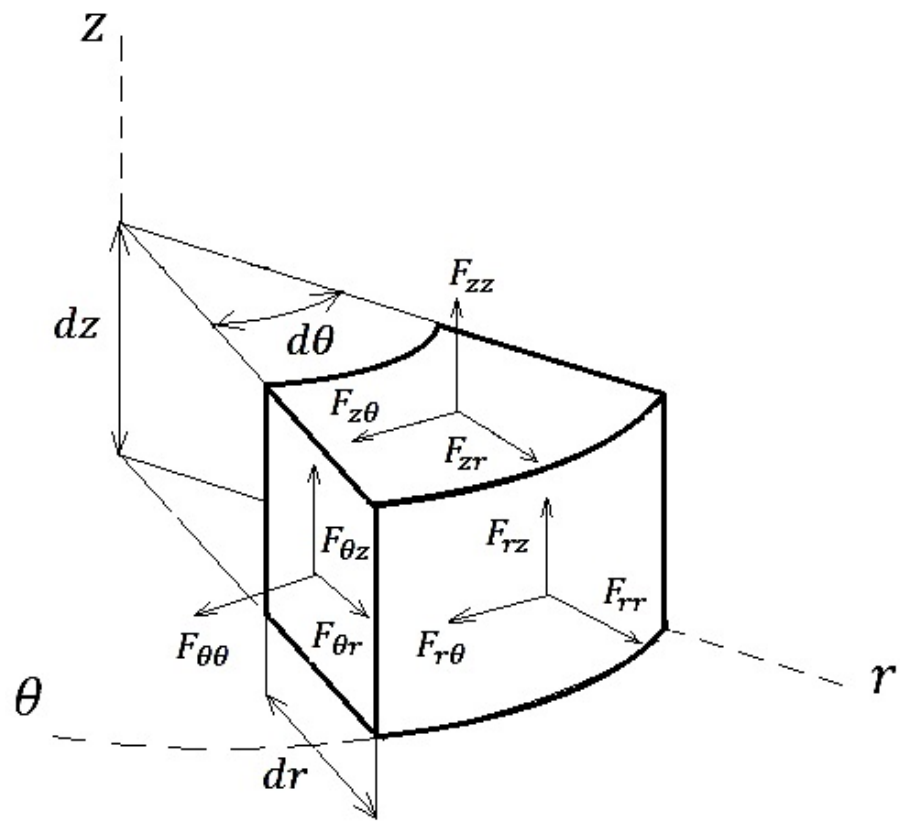

Fig. 2. The operator forces of solid continuous systems in polar coordinates systems

By considering Fig. 2 the equilibrium equations are:

$$
\begin{aligned}
& \sum \overrightarrow{P_{r}}=0 \Rightarrow F_{r r} \hat{e}_{r}+F_{\theta r} \hat{e}_{r}+F_{z r} \hat{e}_{r}+F_{\theta \theta} \frac{\partial \hat{e}_{\theta}}{\partial \theta}+F_{r}=0 \\
& \sum \overrightarrow{P_{\theta}}=0 \Rightarrow F_{r \theta} \hat{e}_{\theta}+F_{\theta \theta} \hat{e}_{\theta}+F_{z \theta} \hat{e}_{\theta}+F_{\theta r} \frac{\partial \hat{e}_{r}}{\partial \theta}+F_{\theta}=0 \\
& \sum \overrightarrow{P_{z}}=0 \Rightarrow F_{r z} \hat{e}_{z}+F_{\theta z} \hat{e}_{z}+F_{z z} \hat{e}_{z}+F_{z}=0
\end{aligned}
$$

The terms as $F_{\theta \theta}\left(\partial \hat{e}_{\theta} / \partial \theta\right)$ and $F_{\theta r}\left(\partial \hat{e}_{r} / \partial \theta\right)$ in this equation are due to the relationship between orthogonal directions in polar coordinates (i.e. $\partial \hat{e}_{\theta} / \partial \theta=-\hat{e}_{r}$ and $\partial \hat{e}_{r} / \partial \theta=\hat{e}_{\theta}$ ). By eliminating $\hat{e}_{r}$, $\hat{e}_{\theta}$ and $\hat{e}_{z}$ from Eq. (8), we have:

$F_{r r}+F_{\theta r}+F_{z r}-F_{\theta \theta}+F_{r}=0$ 
$F_{r \theta}+F_{\theta \theta}+F_{z \theta}+F_{\theta r}+F_{\theta}=0$

$F_{r z}+F_{\theta z}+F_{z z}+F_{z}=0$

Using Eq. (9) in Eq. (2) leads to following equations be achieved:

$\frac{\partial}{\partial r}\left(\frac{\partial^{2} F_{r r}}{\partial \theta \partial z}\right)+\frac{\partial}{\partial \theta}\left(\frac{\partial^{2} F_{\theta r}}{\partial r \partial z}\right)+\frac{\partial}{\partial z}\left(\frac{\partial^{2} F_{z r}}{\partial \theta \partial r}\right)-\left(\frac{\partial^{2} F_{\theta \theta}}{\partial r \partial z}\right)+\frac{\partial^{3} F_{r}}{\partial r \partial \theta \partial z}=0$

$\frac{\partial}{\partial r}\left(\frac{\partial^{2} F_{r \theta}}{\partial \theta \partial z}\right)+\frac{\partial}{\partial \theta}\left(\frac{\partial^{2} F_{\theta \theta}}{\partial r \partial z}\right)+\frac{\partial}{\partial z}\left(\frac{\partial^{2} F_{z \theta}}{\partial \theta \partial r}\right)+\left(\frac{\partial^{2} F_{\theta r}}{\partial r \partial z}\right)+\frac{\partial^{3} F_{\theta}}{\partial r \partial \theta \partial z}=0$

$\frac{\partial}{\partial r}\left(\frac{\partial^{2} F_{r z}}{\partial \theta \partial z}\right)+\frac{\partial}{\partial \theta}\left(\frac{\partial^{2} F_{\theta z}}{\partial r \partial z}\right)+\frac{\partial}{\partial z}\left(\frac{\partial^{2} F_{z z}}{\partial \theta \partial r}\right)+\frac{\partial^{3} F_{z}}{\partial r \partial \theta \partial z}=0$

Inserting Eq. (7) into Eq. (10) results:

$$
\begin{aligned}
& \frac{\partial\left(r \sigma_{r r}\right)}{\partial r}+\frac{\partial\left(\sigma_{\theta r}\right)}{\partial \theta}+\frac{\partial\left(r \sigma_{z r}\right)}{\partial z}-\sigma_{\theta \theta}+r f_{r}=0 \\
& \frac{\partial\left(r \sigma_{r \theta}\right)}{\partial r}+\frac{\partial\left(\sigma_{\theta \theta}\right)}{\partial \theta}+\frac{\partial\left(r \sigma_{z \theta}\right)}{\partial z}+\sigma_{\theta r}+r f_{\theta}=0 \\
& \frac{\partial\left(r \sigma_{r z}\right)}{\partial r}+\frac{\partial\left(\sigma_{\theta z}\right)}{\partial \theta}+\frac{\partial\left(r \sigma_{z z}\right)}{\partial z}+r f_{z}=0
\end{aligned}
$$

By applying derivative operator and some algebraic operations Eq. (11) is converted to below:

$$
\begin{aligned}
& \frac{\partial \sigma_{r r}}{\partial r}+\frac{1}{r} \frac{\partial \sigma_{\theta r}}{\partial \theta}+\frac{1}{r}\left(\sigma_{r r}-\sigma_{\theta \theta}\right)+\frac{\partial \sigma_{z r}}{\partial z}+f_{r}=0 \\
& \frac{\partial \sigma_{r \theta}}{\partial r}+\frac{1}{r} \frac{\partial \sigma_{\theta \theta}}{\partial \theta}+\frac{2}{r} \sigma_{r \theta}+\frac{\partial \sigma_{z \theta}}{\partial z}+f_{\theta}=0 \\
& \frac{\partial \sigma_{r z}}{\partial r}+\frac{1}{r} \sigma_{r z}+\frac{1}{r} \frac{\partial \sigma_{\theta z}}{\partial \theta}+\frac{\partial \sigma_{z z}}{\partial z}+f_{z}=0
\end{aligned}
$$

\subsection{Equilibrium equations in spherical coordinates system}

The stress components in spherical coordinates with referring to Fig. 3 are:

$$
\begin{aligned}
& \sigma_{r r}=\frac{1}{r^{2} \sin \theta} \frac{\partial^{2} F_{r r}}{\partial \theta \partial \varphi} \quad \sigma_{\theta r}=\frac{1}{r \sin \theta} \frac{\partial^{2} F_{\theta r}}{\partial r \partial \varphi} \quad \sigma_{\varphi r}=\frac{1}{r} \frac{\partial^{2} F_{\varphi r}}{\partial r \partial \theta} \quad f_{r}=\frac{1}{r^{2} \sin \theta} \frac{\partial^{3} F_{r}}{\partial r \partial \theta \partial \varphi} \\
& \sigma_{r \varphi}=\frac{1}{r^{2} \sin \theta} \frac{\partial^{2} F_{r \varphi}}{\partial \theta \partial \varphi} \quad \sigma_{\theta \varphi}=\frac{1}{r \sin \theta} \frac{\partial^{2} F_{\theta \varphi}}{\partial r \partial \varphi} \quad \sigma_{\varphi \varphi}=\frac{1}{r} \frac{\partial^{2} F_{\varphi \varphi}}{\partial r \partial \theta} \quad f_{\theta}=\frac{1}{r^{2} \sin \theta} \frac{\partial^{3} F_{\theta}}{\partial r \partial \theta \partial \varphi} \\
& \sigma_{r \theta}=\frac{1}{r^{2} \sin \theta} \frac{\partial^{2} F_{r \theta}}{\partial \theta \partial \varphi} \quad \sigma_{\theta \theta}=\frac{1}{r \sin \theta} \frac{\partial^{2} F_{\theta \theta}}{\partial r \partial \varphi} \quad \sigma_{\varphi \theta}=\frac{1}{r} \frac{\partial^{2} F_{\varphi \theta}}{\partial r \partial \theta} \quad f_{\varphi}=\frac{1}{r^{2} \sin \theta} \frac{\partial^{3} F_{\varphi}}{\partial r \partial \theta \partial \varphi}
\end{aligned}
$$

Equilibrium equations of spherical coordinates in three orthogonal directions are achieved by considering Fig. 3.

$$
\begin{aligned}
& \sum \overrightarrow{P_{r}}=0 \Rightarrow F_{r r} \hat{e}_{r}+F_{\theta r} \hat{e}_{r}+F_{\varphi r} \hat{e}_{r}+F_{\theta \theta} \frac{\partial \hat{e}_{\theta}}{\partial \theta}+F_{\varphi \varphi} \frac{\partial \hat{e}_{\varphi}}{\partial \varphi}+F_{r}=0 \\
& \sum \overrightarrow{P_{\theta}}=0 \Rightarrow F_{r \theta} \hat{e}_{\theta}+F_{\theta \theta} \hat{e}_{\theta}+F_{\varphi \theta} \hat{e}_{\theta}+F_{\theta r} \frac{\partial \hat{e}_{r}}{\partial \theta}+F_{\varphi \varphi} \frac{\partial \hat{e}_{\varphi}}{\partial \varphi}+F_{\theta}=0
\end{aligned}
$$


$\sum \overrightarrow{P_{\varphi}}=0 \Rightarrow F_{r \varphi}+F_{\theta \varphi}+F_{\varphi \varphi}+F_{\varphi r} \frac{\partial \hat{e}_{r}}{\partial \varphi}+F_{\varphi \theta} \frac{\partial \hat{e}_{\theta}}{\partial \varphi}+F_{\varphi}=0$

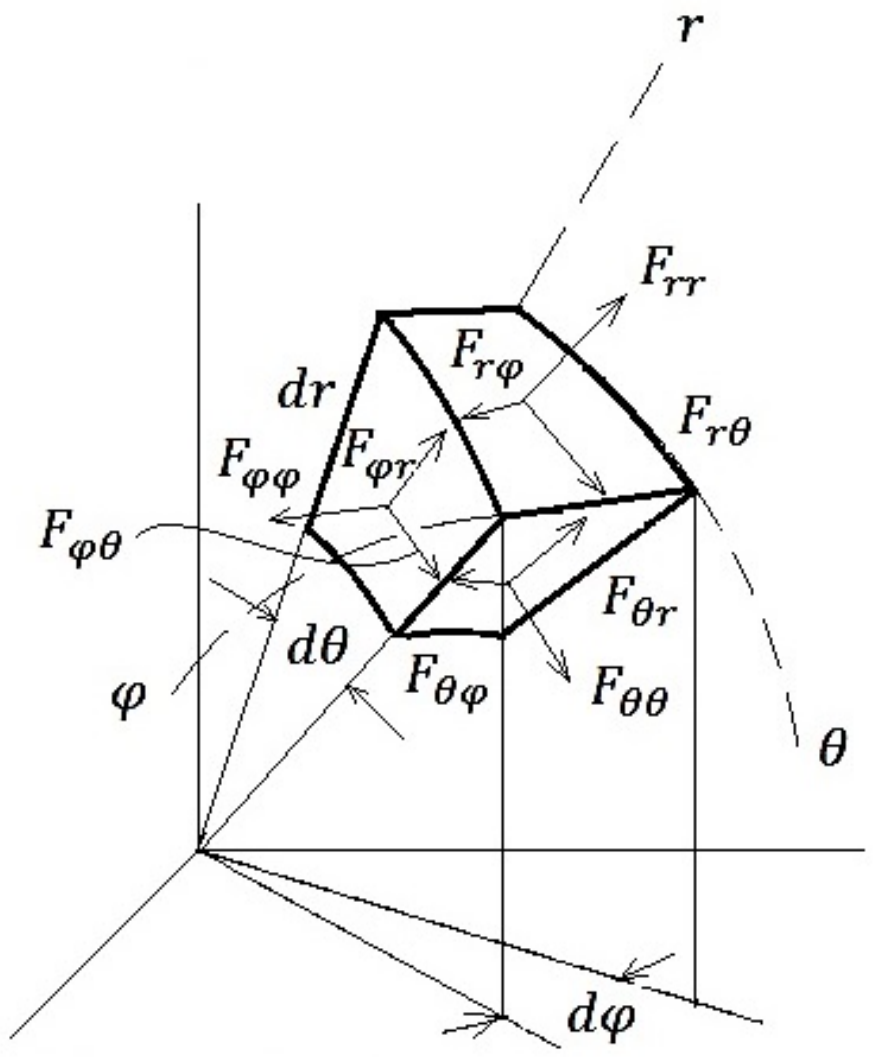

Fig. 3. The potential forces of solid continuous systems in spherical coordinates systems

The variations of unit vectors in spherical coordinates system with respect to other directions are: (i.e. $\quad d \hat{e}_{r}=\left(\hat{e}_{\theta} d \theta+\sin \theta \hat{e}_{\varphi} d \varphi\right), \quad d \hat{e}_{\theta}=\left(-\hat{e}_{r} d \theta+\cos \theta \hat{e}_{\varphi} d \varphi\right)$ and $d \hat{e}_{\varphi}=\left(-\sin \theta \hat{e}_{r} d \varphi-\right.$ $\left.\cos \theta \hat{e}_{\theta} d \varphi\right)$ ). Eliminating $\hat{e}_{r}, \hat{e}_{\theta}$ and $\hat{e}_{\varphi}$, from Eq. (14) gives:

$F_{r r}+F_{\theta r}+F_{\varphi r}-F_{\theta \theta}-F_{\varphi \varphi} \sin \theta+F_{r}=0$

$F_{r \theta}+F_{\theta \theta}+F_{\varphi \theta}+F_{\theta r}-F_{\varphi \varphi} \cos \theta+F_{\theta}=0$

$F_{r \varphi}+F_{\theta \varphi}+F_{\varphi \varphi}+F_{\varphi r} \sin \theta+F_{\varphi \theta} \cos \theta+F_{\varphi}=0$

Replacing Eq. (15) into (2) these equations become:

$\frac{\partial}{\partial r}\left(\frac{\partial^{2} F_{r r}}{\partial \theta \partial \varphi}\right)+\frac{\partial}{\partial \theta}\left(\frac{\partial^{2} F_{\theta r}}{\partial r \partial \varphi}\right)+\frac{\partial}{\partial \varphi}\left(\frac{\partial^{2} F_{\varphi r}}{\partial r \partial \theta}\right)-\frac{\partial^{2} F_{\theta \theta}}{\partial r \partial \varphi}-\frac{\partial^{2} F_{\varphi \varphi}}{\partial r \partial \theta} \sin \theta+\frac{\partial^{3} F_{r}}{\partial r \partial \theta \partial \varphi}=0$

$\frac{\partial}{\partial r}\left(\frac{\partial^{2} F_{r \theta}}{\partial \theta \partial \varphi}\right)+\frac{\partial}{\partial \theta}\left(\frac{\partial^{2} F_{\theta \theta}}{\partial r \partial \varphi}\right)+\frac{\partial}{\partial \varphi}\left(\frac{\partial^{2} F_{\varphi \theta}}{\partial r \partial \theta}\right)+\frac{\partial^{2} F_{\theta r}}{\partial r \partial \varphi}-\frac{\partial^{2} F_{\varphi \varphi}}{\partial r \partial \theta} \cos \theta+\frac{\partial^{3} F_{\theta}}{\partial r \partial \theta \partial \varphi}=0$

$\frac{\partial}{\partial r}\left(\frac{\partial^{2} F_{r \varphi}}{\partial \theta \partial \varphi}\right)+\frac{\partial}{\partial \theta}\left(\frac{\partial^{2} F_{\theta \varphi}}{\partial r \partial \varphi}\right)+\frac{\partial}{\partial \varphi}\left(\frac{\partial^{2} F_{\varphi \varphi}}{\partial r \partial \theta}\right)+\frac{\partial^{2} F_{\varphi r}}{\partial r \partial \theta} \sin \theta+\frac{\partial^{2} F_{\varphi \theta}}{\partial r \partial \theta} \cos \theta+\frac{\partial^{3} F_{\varphi}}{\partial r \partial \theta \partial \varphi}=0$

By using Eq. (13) in Eq. (16), results as following:

$\frac{\partial}{\partial r}\left(r^{2} \sin \theta \sigma_{r r}\right)+\frac{\partial}{\partial \theta}\left(r \sin \theta \sigma_{\theta r}\right)+\frac{\partial}{\partial \varphi}\left(r \sigma_{\varphi r}\right)-r \sin \theta \sigma_{\theta \theta}-r \sin \theta \sigma_{\varphi \varphi}+r^{2} \sin \theta f_{r}=0$ 
$\frac{\partial}{\partial r}\left(r^{2} \sin \theta \sigma_{r \theta}\right)+\frac{\partial}{\partial \theta}\left(r \sin \theta \sigma_{\theta \theta}\right)+\frac{\partial}{\partial \varphi}\left(r \sigma_{\varphi \theta}\right)+r \sin \theta \sigma_{\theta r}-r \cos \theta \sigma_{\varphi \varphi}+r^{2} \sin \theta f_{\theta}=0$

$\frac{\partial}{\partial r}\left(r^{2} \sin \theta \sigma_{r \varphi}\right)+\frac{\partial}{\partial \theta}\left(r \sin \theta \sigma_{\theta \varphi}\right)+\frac{\partial}{\partial \varphi}\left(r \sigma_{\varphi \varphi}\right)+r \sin \theta \sigma_{\varphi r}+r \cos \theta \sigma_{\varphi \theta}+r^{2} \sin \theta f_{\varphi}$

$$
=0
$$

Finally, by simplification of Eq. (17) equilibrium equations in spherical coordinates system are as following:

$\frac{\partial \sigma_{r r}}{\partial r}+\frac{1}{r} \frac{\partial \sigma_{\theta r}}{\partial \theta}+\frac{1}{r \sin \theta}\left(\frac{\partial \sigma_{\varphi r}}{\partial \varphi}\right)+\frac{1}{r}\left(2 \sigma_{r r}-\sigma_{\theta \theta}-\sigma_{\varphi \varphi}+\sigma_{\theta r} \cot \theta\right)+f_{r}=0$

$\frac{\partial \sigma_{r \theta}}{\partial r}+\frac{1}{r} \frac{\partial \sigma_{\theta \theta}}{\partial \theta}+\frac{1}{r \sin \theta}\left(\frac{\partial \sigma_{\theta \varphi}}{\partial \varphi}\right)+\frac{1}{r}\left(3 \sigma_{r \theta}+\left(\sigma_{\theta \theta}-\sigma_{\varphi \varphi}\right) \cot \theta\right)+f_{\theta}=0$

$\frac{\partial \sigma_{r \varphi}}{\partial r}+\frac{1}{r} \frac{\partial \sigma_{\theta \varphi}}{\partial \theta}+\frac{1}{r \sin \theta}\left(\frac{\partial \sigma_{\varphi \varphi}}{\partial \varphi}\right)+\frac{1}{r}\left(3 \sigma_{r \varphi}+2 \sigma_{\theta \varphi} \cot \theta\right)+f_{\varphi}=0$

where, exact agreement can be seen in the results of method (Saad 2005). Basied on new method and for more clarity of the concept, derivations of heat equation are given in the appendix.

\section{Nonlinear case}

In this section general form of nonlinear governing equations in Cartesian coordinates is derived. In the linear conditions, each surface of element involves only one component of stress in each direction. For example in a surface with the normal unite vector of $x$, only $\sigma_{x z}$ applies in the $z$ direction. While for the nonlinear conditions, because of large slopes, there are three components of stresses in each direction. This issue is shown in Fig. (4). As can be observed $\sigma_{x x} \partial w / \partial x+$ $\sigma_{x y} \partial w / \partial y+\sigma_{x z}$ are the stress components in $z$ direction.

This matter can be reviewed by comparing Euler and Lagrange configurations in Fig. (4). Origin of coordinates system in the Euler and Lagrange views are $x^{*}, y^{*}, z^{*}$ and $x, y, z$, respectively and are coincided on each other before deformation. As the Lagrangian coordinates is the original one, so the stress field of moving points should be explained based on its variables, too. Amabili (2008) extracted the relationship between Lagrangian and Eulerian stress components versus $u_{i}(u, v, w$ in $x, y$ and $z$ directions respectively) the deformations of continuous systems and their derivatives. Thus, for nonlinear case the potential forces $F_{i j}$, can be defined versus a combination of linear and nonlinear terms, as following:

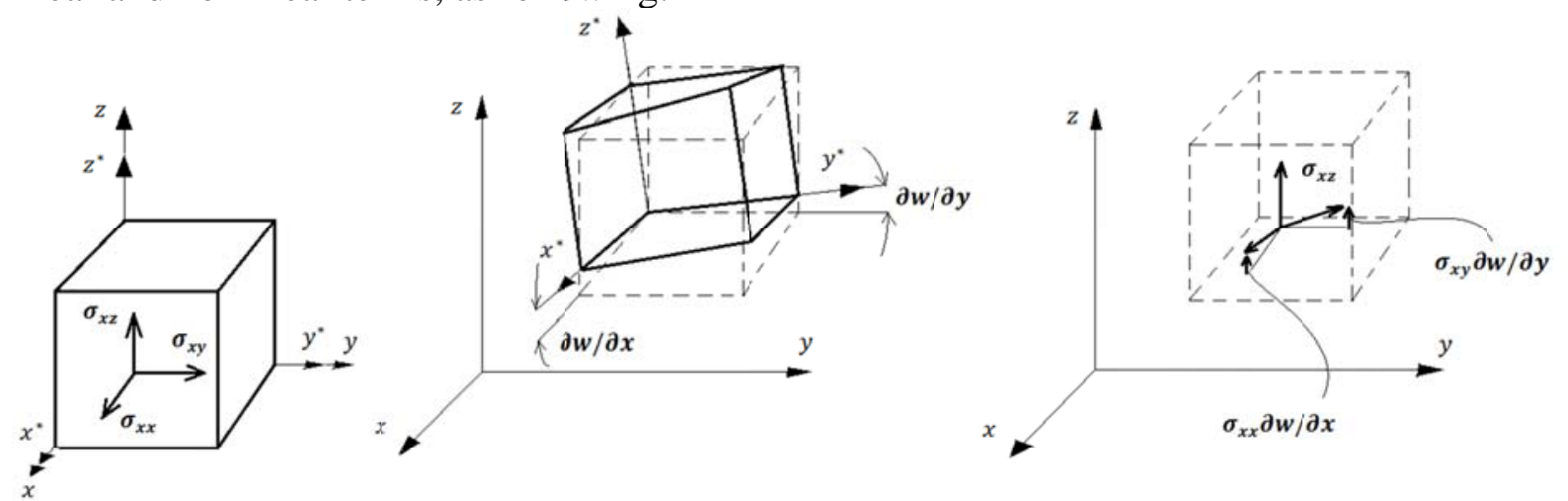

Linear condition

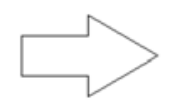

large deformation

Non linear condition

Fig. 4. Effect of $\sigma_{x j}$ components in $z$ direction in the linear and nonlinear case 
$\frac{\partial^{2} F_{x x}}{\partial y \partial z}=\sigma_{x x}+\sigma_{x y} \frac{\partial u}{\partial y}+\sigma_{x z} \frac{\partial u}{\partial z}$

$\frac{\partial^{2} F_{x z}}{\partial y \partial z}=\sigma_{x x} \frac{\partial w}{\partial x}+\sigma_{x y} \frac{\partial w}{\partial y}+\sigma_{x z}$

$\frac{\partial^{2} F_{y x}}{\partial x \partial z}=\sigma_{y x}+\sigma_{y y} \frac{\partial u}{\partial y}+\sigma_{y z} \frac{\partial u}{\partial z}$

$\frac{\partial^{2} F_{y z}}{\partial x \partial z}=\sigma_{y x} \frac{\partial w}{\partial x}+\sigma_{y y} \frac{\partial w}{\partial y}+\sigma_{y z}$

$\frac{\partial^{2} F_{z x}}{\partial y \partial x}=\sigma_{z x}+\sigma_{z y} \frac{\partial u}{\partial y}+\sigma_{z z} \frac{\partial u}{\partial z}$

$\frac{\partial^{2} F_{z z}}{\partial y \partial x}=\sigma_{z x} \frac{\partial w}{\partial x}+\sigma_{z y} \frac{\partial w}{\partial y}+\sigma_{z z}$

$$
\begin{aligned}
& \frac{\partial^{2} F_{x y}}{\partial y \partial z}=\sigma_{x x} \frac{\partial v}{\partial x}+\sigma_{x y}+\sigma_{x z} \frac{\partial v}{\partial z} \\
& f_{x}=\frac{\partial^{3} F_{x}}{\partial x \partial y \partial z} \\
& \frac{\partial^{2} F_{y y}}{\partial x \partial z}=\sigma_{y x} \frac{\partial v}{\partial x}+\sigma_{y y}+\sigma_{y z} \frac{\partial v}{\partial z} \\
& f_{y}=\frac{\partial^{3} F_{y}}{\partial x \partial y \partial z} \\
& \frac{\partial^{2} F_{z y}}{\partial y \partial x}=\sigma_{z x} \frac{\partial v}{\partial x}+\sigma_{z y}+\sigma_{z z} \frac{\partial v}{\partial z} \\
& f_{z}=\frac{\partial^{3} F_{z}}{\partial x \partial y \partial z}
\end{aligned}
$$

Result of inserting Eq. (19) in Eq. (5) is as below:

$$
\begin{aligned}
& \frac{\partial}{\partial x}\left(\sigma_{x x}+\sigma_{x y} \frac{\partial u}{\partial y}+\sigma_{x z} \frac{\partial u}{\partial z}\right)+\frac{\partial}{\partial y}\left(\sigma_{y x}+\sigma_{y y} \frac{\partial u}{\partial y}+\sigma_{y z} \frac{\partial u}{\partial z}\right)+\frac{\partial}{\partial z}\left(\sigma_{z x}+\sigma_{z y} \frac{\partial u}{\partial y}+\sigma_{z z} \frac{\partial u}{\partial z}\right) \\
& +f_{x}=0 \\
& \frac{\partial}{\partial x}\left(\sigma_{x x} \frac{\partial v}{\partial x}+\sigma_{x y}+\sigma_{x z} \frac{\partial v}{\partial z}\right)+\frac{\partial}{\partial y}\left(\sigma_{y x} \frac{\partial v}{\partial x}+\sigma_{y y}+\sigma_{y z} \frac{\partial v}{\partial z}\right)+\frac{\partial}{\partial z}\left(\sigma_{z x} \frac{\partial v}{\partial x}+\sigma_{z y}+\sigma_{z z} \frac{\partial v}{\partial z}\right) \\
& +f_{y}=0 \\
& \frac{\partial}{\partial x}\left(\sigma_{x x} \frac{\partial w}{\partial x}+\sigma_{x y} \frac{\partial w}{\partial y}+\sigma_{x z}\right)+\frac{\partial}{\partial y}\left(\sigma_{y x} \frac{\partial w}{\partial x}+\sigma_{y y} \frac{\partial w}{\partial y}+\sigma_{y z}\right)+\frac{\partial}{\partial z}\left(\sigma_{z x} \frac{\partial w}{\partial x}+\sigma_{z y} \frac{\partial w}{\partial y}+\sigma_{z z}\right) \\
& +f_{z}=0 \text {. }
\end{aligned}
$$

Last equation has not been expanded for abbreviation. In this equation if slope of deformations in $x$ and $y$ directions (slopes of $u$ and $v$ ) be ignored, governing equations of transverse vibration in mechanical structures will be achieved. Because, $\partial u / \partial x_{i}$ and $\partial v / \partial x_{i}$ are negligible in comparison with $\partial w / \partial x_{i}$ for transverse deformation of mechanical structures. While Eq. (20) has a general form and is extendable to any arbitrary problem.

\section{Conclusion}

The concept of governing equations in continuous systems was reviewed. It was shown that, for continuous systems the motions equations should be expandable to all points of system. Because a physical particle cannot be a mathematical point, so there is a challenge in the concept of governing equations in continuous systems which guided us toward a fundamental investigation about these systems. So, it was shown that using from intensive properties generalizes the governing equations of a particle with specified dimensions to any internal dimensionless point of it. Basics of a simple method were established for deriving the governing equations in continuous systems based on applying intensive properties. By extraction of equilibrium equations in Cartesian, polar and spherical coordinates systems, the method is verified and exact agreement was observed. The new method is more efficient in comparison with old methods such as Hamiltonian and classic methods due to its simplicity. Other methods contain a hard task in their solution process and needs more time. Moreover of saving time in new method, it has made possible to be realized concept of governing equations in continuous systems for researchers. Direct method is also extensible to other equations and a united procedure has been offered for extracting the governing equations in continuous systems. 


\section{References}

Amabili, M. (2008). Nonlinear vibrations and stability of shells and plates. Cambdrage University Press, New York, USA

Saad, M.H. (2005). Elasticity Theory, Applications, and Numerics $\left(2^{\text {nd }} e d.\right)$. Elsavier Inc

\section{Appendix}

For derivation of heat equation for a homogenous material as a solid continuous system, direct method is used as follows:

First law of thermodynamics:

$Q=Q_{x}+Q_{y}+Q_{z}=m c \frac{\partial T}{\partial t}$

Fourier equations:

$\frac{\partial^{2} Q_{x}}{\partial y \partial z}=K \frac{\partial T}{\partial x} \frac{\partial^{2} Q_{y}}{\partial x \partial z}=K \frac{\partial T}{\partial y} \frac{\partial^{2} Q_{z}}{\partial x \partial y}=K \frac{\partial T}{\partial z}$

Direct method:

$\frac{\partial}{\partial x}\left(\frac{\partial^{2} Q_{x}}{\partial y \partial z}\right)+\frac{\partial}{\partial y}\left(\frac{\partial^{2} Q_{y}}{\partial x \partial z}\right)+\frac{\partial}{\partial z}\left(\frac{\partial^{2} Q_{z}}{\partial x \partial y}\right)=\frac{\partial}{\partial V} m c \frac{\partial T}{\partial t}$

Inserting Eq. (A.2) in (A.3) results heat equation as following:

$\frac{\partial^{2} T}{\partial x^{2}}+\frac{\partial^{2} T}{\partial y^{2}}+\frac{\partial^{2} T}{\partial z^{2}}=\frac{\rho c}{K} \frac{\partial T}{\partial t}$ 